\title{
Statistical Tolerance Analysis Based on Beta Distributions
}

\author{
Shui-Shun Lin, National Chinyi Institute of Technology, Taichung, Taiwan \\ Hsu-Pin (Ben) Wang and Chun (Chuck) Zhang, Florida A\&M University/Florida State University, \\ Tallahassee, Florida
}

\begin{abstract}
Statistical tolerance analysis is being studied extensively. Normal distributions have been traditionally assumed in tolerance analysis; however, little evidence has been found to support that assumption in the real world. In fact, beta distributions are found to be suited for modeling manufacturing processes because of the flexibility in fitting various shapes of distribution. In this paper, a method called the beta distribution approximation method (BDAM) is developed and applied to solve tolerance analysis problems. In the formulation of the BDAM, a beta distribution is employed to model a manufacturing process. The resultant distribution obtained from adding up a number of beta distributions is approximated with a beta distribution. A validation process of the BDAM is carried out and the results are found to be promising. An example is provided to illustrate the BDAM application. Observations are made to conclude the paper.
\end{abstract}

Keywords: Statistical Tolerance Analysis, Tolerance Synthesis, Beta Distributions, Distribution Approximation

\section{Introduction}

Tolerance analysis and synthesis is traditionally studied in a worst-case context. ${ }^{1}$ Tolerance calculations are conducted based on the extreme conditions of a production error distribution. This approach is mathematically simple but may lead to unnecessarily tight tolerances, which increase manufacturing costs. Because the dimensional variations of machined parts (other than single-piece production) naturally follow statistical distributions, the chance of a part being manufactured at the extreme dimension is usually small. Thus, statistical tolerancing methods make more sense as far as economics is concerned.

Statistical tolerancing has been studied for decades. Many assembly statistical tolerancing models have been proposed and investigated. Mansoor proposed a formulation of statistical tolerance calculation based on a normal distribution. ${ }^{2}$ Parkinson modified it in $1984 .{ }^{3}$ Based on their assumptions, the tolerance range of the resultant dimension was calculated by the root sum square (RSS) method.
The RSS calculation was developed based on an assumption that the resultant dimension followed a normal distribution. In real production, this assumption may not be valid. In fact, many component dimensions do not follow a normal distribution. Asymmetric distributions are often introduced by system errors, and small-dimension chains are common. Significant errors may be introduced by this unrealistic formulation. ${ }^{4}$

Modifications to handle asymmetric production distributions were introduced. Spotts' modified model was used to describe the distribution of resultant dimensions more accurately by combining the worst-case and statistical approaches. ${ }^{5}$ Bjorke studied a beta distribution model for the resultant dimension. ${ }^{6}$ However, the same root sum square formulation obtained from the normal distribution assumption was used in their non-normal distribution methods.

Greenwood and Chase introduced a modified sum of squares method that increased the simple RSS tolerance sum by a correction factor. ${ }^{4}$ Many other correction factors were suggested. However, this approach had a serious limitation: when the number of components in the assembly was two, the modified sum of squares assembly tolerance was even greater than that obtained from the worst-case method. Greenwood and Chase also introduced a unified tolerance analysis method based on the estimated mean shift model. This method was oriented toward component dimensional distribution. It could be used to deal with different part dimensions with a skewed distribution and to incorporate manufacturing data related to different component dimensions into a design. However, this method could only accommodate a mean shift symmetrical to the nominal dimension. Furthermore, the determination of the mean shift factor might be very difficult. ${ }^{\text {? }}$

While it is useful under some conditions, normal distribution approximations and simple statistical 
approaches do present serious limitations. Several studies have been conducted to solve this problem. The tolerancing methodology developed by Bjorke overcomes some of the difficulties associated with the normal distribution assumption. ${ }^{6}$ This study focused on the resultant distribution only, and a beta distribution was used to describe the resultant of a combination of stochastic variables. The component distributions were not given any treatment.

Dong introduced a new tolerance calculation approach, range-truncation, to deal with statistical tolerancing based on component dimension distributions rather than the resultant dimension distribution. ${ }^{8}$ The resultant dimension distribution was not studied-only the range was of concern. This approach took into account the statistical consideration in the calculation of the truncated distribution range for each component distribution. However, the calculation of the resultant tolerance was based on a given confidence level and was performed using the worst-case approach. This approach lacked the ability to handle tolerance analysis with manufacturing yield prediction.

For statistical tolerance analysis, characterization of the process distribution is a prerequisite. Traditional tolerance calculations are based on the assumption of normal distributions. In practice, process distributions are found skewed and/or with process mean shift. This may be caused by tool wear or setup errors. Researchers have been attempting to model the process distribution for tolerance analysis and synthesis. In this paper, a beta distribution approximation method is introduced to deal with tolerance calculation for non-normally distributed processes.

\section{Beta Distribution}

Beta distributions have been chosen for this work because they satisfy the following requirements: (1) the capability of modeling various distribution shapes, such as those generated by random errors, random distributions with changing standard deviations, or random distributions with changing expected values; (2) the capability of supporting the calculation of an inverse probability distribution function, which determines the distribution value corresponding to a given confidence level; and (3) the capabil-

* As opposed to a normal distribution, which extends infinitive to both sides of the distribution. ity of modeling distributions with a finite range.*

The beta distribution has been used in the past for various engineering problems. ${ }^{9}$ A variety of distribution shapes can be modeled with only two shape parameters, $\alpha$ and $\beta$, as illustrated in Figure 1 .

The beta distribution is defined over the interval $(0,1)$. To differentiate it from a real distribution over a finite interval $(a, b)$, it is called the unit beta distribution. In this paper, $x$ denotes the generalized distribution variable over the range of $(a, b)$, while $u$ denotes the unit beta distribution variable over the range of $(0,1)$.

The unit beta probability density function is given as follows:

$$
f(u ; \alpha, \beta)=\left\{\begin{array}{l}
\frac{\Gamma(\alpha+\beta)}{\Gamma(\alpha) \Gamma(\beta)} u^{\alpha-1}(1-u)^{\beta-1}, 0 \leq u \leq 1,0 \leq \alpha, 0 \leq \beta \\
0, \text { elsewhere }
\end{array}\right.
$$

The unit beta probability distribution function is defined as:

$$
F(u ; \alpha, \beta)=\left\{\begin{array}{l}
0, \mathrm{u}<0 \\
\frac{\Gamma(\alpha+\beta)}{\Gamma(\alpha) \Gamma(\beta)} \int_{0}^{u} t^{\alpha-1}(1-t)^{\beta-1} d t, 0 \leq u \leq 1 \\
1, u>1
\end{array}\right.
$$

$F(U ; \alpha, \beta)$ is the probability (or confidence level) that $u$ falls within the interval $(0, U)$. $U$ is a constant between 0 and 1 . The probability can be calculated

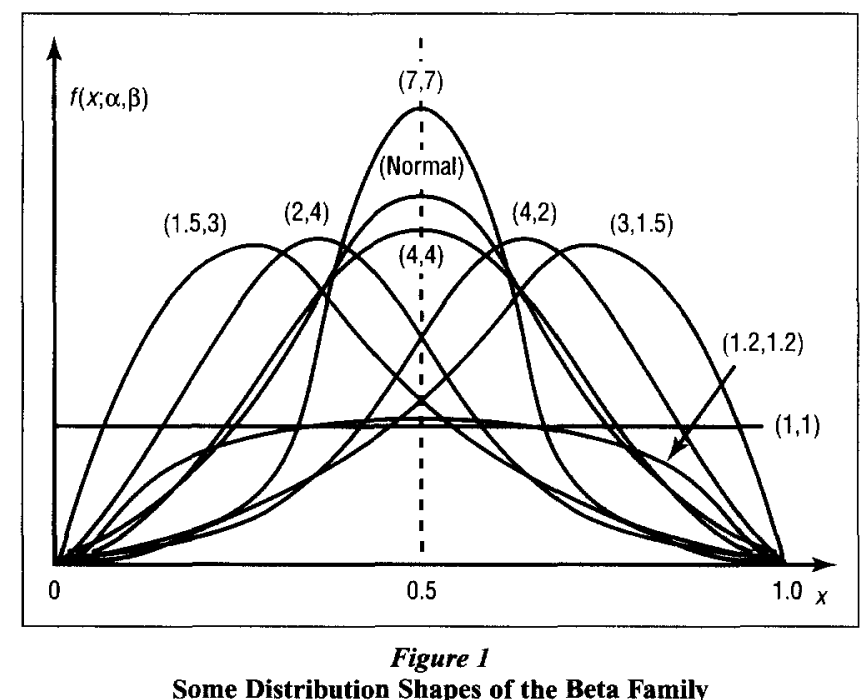


given the two distribution parameters, $\alpha$ and $\beta$, and the distribution range $U$. On the other hand, the distribution range $U$ can be calculated through the inverse integration of the probability distribution function if the distribution parameters, $\alpha$ and $\beta$, and the confidence level are known.

The mean value $(\mu)$ and variance $(v)$ of a unit beta distribution can be calculated from the following equations:

$$
\mu=\frac{\alpha}{\alpha+\beta} \quad v=\frac{\alpha \beta}{(\alpha+\beta)^{2}(\alpha+\beta+1)}
$$

A unit beta random variable $u$ over the range of $(0,1)$ can be rescaled and reallocated to obtain a beta random variable $x$ over the interval $(a, b)$ of the same shape by the transformation:

$$
x=a+(b-a) u
$$

\section{The Beta Distribution Approximation Method}

The beta distribution approximation method (BDAM) was formulated and a series of tests was performed to validate the approximation. Results of the beta distribution approximation are found to be promising. In the following, the beta distribution approximation method is described, as well as its validation process.

\section{Needs for the BDAM}

The distribution of a resultant dimension $X_{r}$ depends on the distributions of the component dimensions $X_{i},(i=1,2, \ldots, n)$. The distribution of $X_{r}$ may therefore be an unknown distribution.

For a tolerance analysis problem, to find the manufacturing yield* and the tolerance range, the resultant dimension distribution should be defined first. There are three approaches to find the resultant distributions: (1) analytical method, (2) simulation, and (3) approximation.

\section{Analytical Method}

An example of an analytical method is the root sum square (RSS) based on the assumption of all component dimensions following a normal distribution. ${ }^{2,10,11}$ It is assumed that component dimensions are normally distributed with known means and standard deviations. The nominal value of the resul- tant dimension is then calculated by summing up the mean value with respect to its direction in the dimension chain; the standard deviation is obtained by using the RSS calculation. Various models are described in the literature review section.

Another analytical method is the convolution operation applied to two independent distributions to find the addition of these two distributions. In principle, one can convolve as many independent random variables as desired. In reality, however, performing the integration alone can be very difficult to carry out. ${ }^{10}$ From the viewpoint of statistics, this convolution operation is tricky to perform. It is not a simple matter of plugging numbers into a formula and performing the calculation. ${ }^{12}$ Because of its complexity, especially for the cases of more than two distributions, the convolution operation is seldom used in tolerance analysis and tolerance synthesis problems.

\section{Simulation}

A commonly used method is Monte Carlo simulation. This method can be applied to either normal distributions, non-normal distributions, or any combinations. Monte Carlo simulation has been found accurate; however, it has major disadvantages in that it requires intensive computation and is very timeconsuming. ${ }^{13}$

\section{Approximation}

Approximation is necessary in the calculation of the resultant dimension distribution when neither the analytical method nor simulation is adequate.

An approximation is needed to carry out the calculation of the resultant dimension when component dimensions are described with beta distributions. This is because a non-normal distribution is assumed. It is known that, from the statistical point of view, the resultant dimension follows a normal distribution if every component dimension is a normal distribution. This relationship does not exist for non-normal distributions. $\dagger$ Furthermore, it has been realized that the simulation approach is

\footnotetext{
* Yield is defined as the percentage of manufactured parts conforming to design specifications.

$\uparrow$ From the viewpoint of statistics, in the case where the number of components is sufficiently large, the resultant dimensions will follow a normal distribution regardless of component dimension distributions.
} 
computationally intensive and, thus, is not efficient if optimization procedures are combined with a simulation approach.

\section{Principle of the BDAM}

It is known that the distribution of the resultant dimension, the sum of many beta distributions, is an unknown distribution. It may be a symmetric distribution or a skewed distribution depending on component dimension distributions. An approximation method is developed to describe this unknown distribution for the purpose of tolerance analysis. The reader will see later that the beta distribution adequately approximates the unknown resultant distribution.

The principle of the distribution approximation method is derived on the basis of a statistical theorem, which is described as follows.

Let $X_{1}, X_{2}, \ldots, X_{n}$ be $n$ random variables, and each of them has a continuous distribution. If the $X_{i}$ 's $(i=1, \ldots, n)$ are independent of each other, the following relationships exist:

$$
\begin{aligned}
& E\left(X_{1}+X_{2}+\ldots+X_{n}\right)=E\left(X_{1}\right)+E\left(X_{2}\right)+\ldots+E\left(X_{n}\right) \\
& \operatorname{Var}\left(X_{1}+X_{2}+\ldots+X_{n}\right)=\operatorname{Var}\left(X_{1}\right)+\operatorname{Var}\left(X_{2}\right)+\ldots+ \\
& \operatorname{Var}\left(X_{n}\right)
\end{aligned}
$$

Assume that the dimension of a component is a random variable and that it follows a beta distribution with parameters $\alpha$ and $\beta$. Each component is fabricated either on a different machine, by a different operator, or at a different time; thus, dimensions of components can be considered independent of each other.

The purpose of the BDAM calculation is to find the parameters of the approximated resultant dimension distribution. Through the following steps, the approximated resultant dimension can be obtained.

1. Given the component dimension distribution parameters $\alpha_{i}, \beta_{i}(i=1, \ldots, n)$, calculate the expected value, $\mu_{i}$, of each component.

2. Calculate the variance $v_{i}$ of each component dimension.

3. Let $\mu_{r}$ be the expected value of the resultant dimension and $v_{r}$ be the variance of the resultant dimension; $\mu_{r}$ and $v_{r}$ can be obtained, that is,

$$
\mu_{r}=\sum_{i=1}^{n} \mu_{i} \quad v_{r}=\sum_{i=1}^{n} v_{i}
$$

4. The parameters of the resultant dimension distribution, $\alpha_{r}$ and $\beta_{r}$, can be obtained by solving two equations with two unknowns and are shown as follows.

$$
\begin{gathered}
\alpha_{r}=\frac{\bar{\mu}_{r}^{2}-\bar{\mu}_{r}^{3}-\bar{\mu}_{r} v_{r}^{\prime}}{v_{r}^{\prime}} \\
\beta_{r}=\frac{1-\bar{\mu}_{r}}{\bar{\mu}_{r}} \cdot \alpha_{r}=\frac{\left(1-\bar{\mu}_{r}\right)\left(\bar{\mu}_{r}^{2}-\bar{\mu}_{r}^{3}-\bar{\mu}_{r} v_{r}^{\prime}\right)}{\bar{\mu}_{r} v_{r}^{\prime}}
\end{gathered}
$$

where:

$$
\bar{\mu}_{r}=\frac{1}{n} \mu_{r}=\frac{1}{n} \sum_{i=1}^{n} \mu_{i} \quad v_{r}^{\prime}=\frac{1}{n^{2}} v_{r}=\frac{1}{n^{2}} \sum_{i=1}^{n} v_{i}
$$

$\bar{\mu}_{r}$ and $v_{r}^{\prime}$ are normalized mean value and variance, respectively, of the unit beta distribution for the resultant dimension.

\section{Validation of the BDAM}

To evaluate the beta distribution approximation method, a validation process has been defined and implemented. The validation process utilizes a random number generator found in a commercial statistics software.* The random number generator is capable of generating various distributions with required distribution parameters; for instance, the mean and standard deviation for a normal distribution.

There are several cases that need to be considered before the validation process is performed. It is essential that the validation covers as many cases of real production processes as possible. Based on this consideration, production processes are classified into three groups: (I) distributions with randomly generated parameters, (II) distributions with left (or negative) skewed parameters, and (III) distributions with right (or positive) skewed parameters. To be more specific, the parameters of distributions in Group I were generated randomly within a certain interval. No constraints are applied to those parameters. The parameters of distributions in Group II were generated the same way as those in Group I,

* MINITAB, from MINITAB, Inc., was utilized and several macros were written to perform the validation process. 
except that only parameters that caused the distribution to be left-skewed were accepted. Likewise, the parameters of distributions in Group III were generated using parameters that caused the distribution to be right-skewed. The first group distributions mimic the common production condition, while the second and third groups handle situations where a mean shift in production occurs.

The validation steps are as follows:

1. Define validation process parameters.

The number of the component distributions, $N$, is set to $2,3,4,5$, and 6 in sequence. Results from a number larger than 6 are found similar to that of 6 . The number of data points for each distribution, $M$, is set to 3000 . The maximum number of validation runs, $L$, is set to 100 . The settings of $M$ and $L$ far exceed the level required to provide enough statistical confidence.

2. Initialize the number of component distribution: set $N=2$.

3 . Generate $2 \times N$ seeds, in the range of $(2.0,8.0)$.

These seeds are the parameters of the component beta distributions. For Group II distributions, these seeds are generated to meet the leftskewed arrangement. For a beta distribution with parameters $\alpha$ and $\beta$, left-skewed arrangement means $\alpha>\beta$. In contrast, for Group III distribution, the parameters are generated for $\alpha<\beta$.

4. Set the counter of runs for each $N$ : set $i=1$.

5. Generate $N$ data sets of beta distributions using the random number generator.

For each data set, the number of points was $M$ $=3000$.

6. Sum up the corresponding data points in each data set and obtain a resultant distribution, $D_{0}$, with 3000 data points.

7. Calculate the normalized distribution by dividing the values of data points by $N$.

The purpose of normalization is to maintain the distribution range within $(0,1)$ so that the beta distribution parameters can be found.

8. Find the normalized sample mean and standard deviation.

9. Generate a beta distribution, $D_{1}$, with parameters derived from the sample mean and sample standard deviation. Calculate the least sum of squares error $\left(E_{1}\right)$ from $D_{1}$ and $D_{0}$.

10. Generate a beta distribution, $D_{2}$, with parameters derived from the BDAM. Calculate the least sum of squares error $\left(E_{2}\right)$ from $D_{2}$ and $D_{0}$.

11. Generate a normal distribution, $D_{3}$, from the sample mean and sample standard deviation. Calculate the least sum of squares error $\left(E_{3}\right)$ from $D_{3}$ and $D_{0}$.

12. Generate a normal distribution, $D_{4}$, with parameters derived from the BDAM. Calculate the least sum of squares error $\left(E_{4}\right)$ from $D_{4}$ and $D_{0}$.

13. Generate a normal distribution, $D_{5}$, with centered mean and sample standard deviation. Calculate the least sum of squares error $\left(E_{5}\right)$ from $D_{5}$ and $D_{0}$.

14. Generate a normal distribution, $D_{6}$, with centered mean and standard deviation derived from the BDAM. Calculate the least sum of squares error $\left(E_{6}\right)$ from $D_{6}$ and $D_{0}$.

15. Record the $E_{i}$ 's for later calculation. Add one (1) to the number of runs counter $i$.

16. If $i<L$, that is, more runs are needed, then go to step 5. Otherwise, increase $N$ by one (1) and check if $N>6$.

17. If the validation process is completed $(N>6)$, average the $E_{i}$ 's and end the process; otherwise, go to step 3 .

The validation process was performed on a 486 PC, and the total elapsed time is 7.50 hours for all distribution groups. The results of this study are tabulated in Tables 1, 2, and 3 on the following pages.

The columns of approximation types are entitled 'beta-s', 'beta-d', 'norm-s', 'norm-d', 'norm-cs', and 'norm-cd'. The prefix 'beta' means that the errors are introduced by using beta distributions to approximate the resultant distribution, while 'norm' stands for approximations using normal distributions. The suffix ' $s$ ' indicates that distribution parameters are computed from distribution data samples, while ' $d$ ' is for parameters derived from the BDAM. The suffix ' $c$ ' indicates a centered mean. Referring to the step-by-step description of the validation process, those values in their corresponding columns are $E_{1}$ 's, $E_{2}$ 's, $E_{3}$ 's, $E_{4}$ 's, $E_{5}$ 's, and $E_{6}$ 's, respectively, and were obtained from the least sum of squares errors of distribution $D_{0}$ versus $D_{1}, D_{2}$, $D_{3}, D_{4}, D_{5}$, and $D_{6}$, respectively.

Tables 1,2 , and 3 show the least sum of squares errors for Group I, II, and III distributions, respec- 
Table 1

Least Sum of Squares Errors (Group I Distributions)

\begin{tabular}{ccccccc}
\hline \multirow{2}{*}{$\begin{array}{c}\text { Number of } \\
\text { Components }\end{array}$} & beta-s & beta-d & norm-s & norm-d & norm-cs & norm-cd \\
\hline 2 & 0.070 & 0.093 & 0.088 & 0.094 & 26.510 & 26.535 \\
3 & 0.039 & 0.057 & 0.095 & 0.103 & 30.142 & 30.204 \\
4 & 0.031 & 0.041 & 0.032 & 0.051 & 6.784 & 6.833 \\
5 & 0.017 & 0.027 & 0.018 & 0.029 & 1.658 & 1.581 \\
6 & 0.017 & 0.027 & 0.019 & 0.028 & 0.200 & 0.207 \\
\hline
\end{tabular}

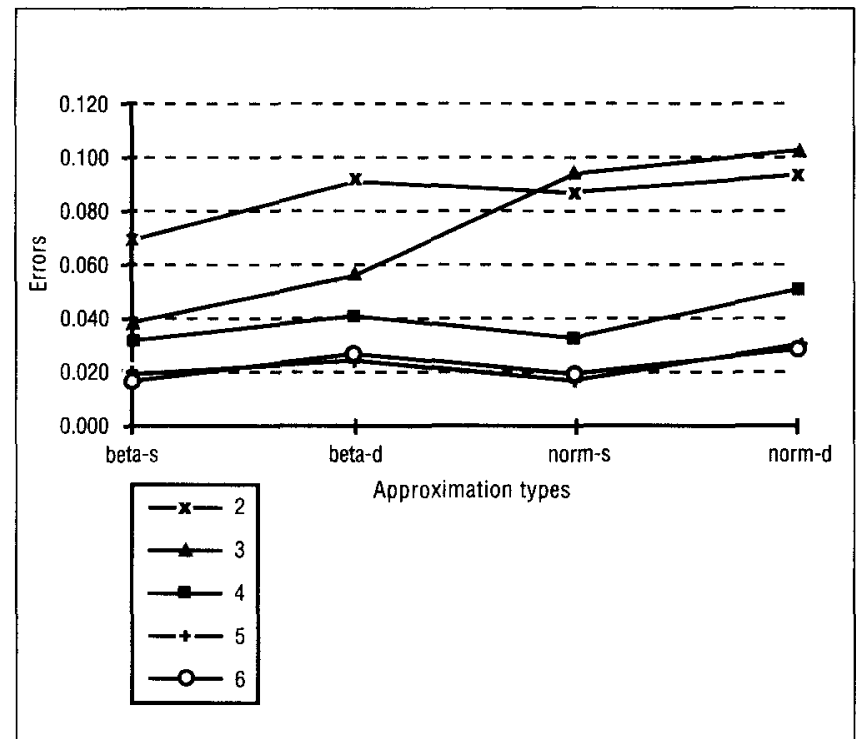

Figure 2

Plot of Approximation Errors Versus Approximation Types (Group I Distributions)

tively. Figures 2, 3, and 4 illustrate the plots of approximation errors versus approximation types, except 'norm-cs' and 'norm-cd' because of their large errors. It is obvious that the approximation errors from a normal distribution with centered mean, labeled 'norm-cs' and 'norm-cd', are relatively larger than that of the others. Comparing the first four columns of the approximation types, 'beta-s', 'beta-d', 'norm-s', and 'norm-d', the difference in the approximation errors for each type is very close when using either five or six components for both. This means that when the component number is greater than five, both beta and normal distributions will obtain close approximation accuracy. In practice, when the number of components is larger than five, the resultant dimension distribution is a normal distribution, regardless of the distributions of the components. ${ }^{13}$ This finding is consistent with other studies found in open literature.
Table 2

Least Sum of Squares Errors (Group II Distributions)

\begin{tabular}{ccccccc}
\hline \multirow{2}{*}{$\begin{array}{c}\text { Number of } \\
\text { Components }\end{array}$} & beta-s & beta-d & norm-s & norm-d & norm-cs & norm-cd \\
\hline 2 & 0.053 & 0.051 & 0.198 & 0.228 & 44.297 & 43.974 \\
3 & 0.042 & 0.058 & 0.121 & 0.134 & 38.469 & 38.462 \\
4 & 0.043 & 0.056 & 0.076 & 0.107 & 32.494 & 32.903 \\
5 & 0.025 & 0.034 & 0.050 & 0.055 & 64.369 & 64.026 \\
6 & 0.023 & 0.029 & 0.034 & 0.043 & 34.121 & 34.081 \\
\hline
\end{tabular}

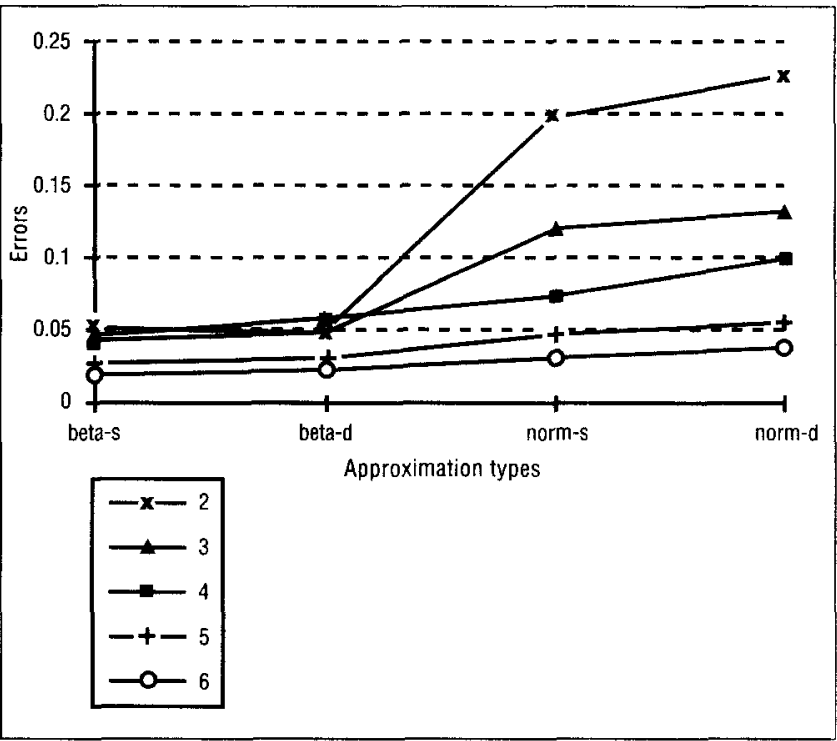

Figure 3

Plot of Approximation Errors Versus Approximation Types (Group II Distributions)

Another observation is that the least sum of squares errors obtained from a beta distribution with parameters derived from a sample mean and sample standard deviation are the smallest, in most cases (see columns labeled 'beta-s'). This observation reveals that the beta approximation using a sample mean and standard deviation appears to be the best approximation. Because this approximation needs a sample mean and standard deviation, and is similar to the Monte Carlo simulation method, it is less suitable if combined with an optimization procedure in tolerance synthesis problems. The reason is that it requires a great deal of computational time. The next best choice, then, is the BDAM, which is labeled 'beta-d'. From the results shown in the tables, errors from the BDAM are generally smaller than those of normal distributions (labeled 'norm-s' and 'norm-d'), especially in the cases of Group II and III distributions. This implies that the BDAM 
Table 3

Least Sum of Squares Errors (Group III Distributions)

\begin{tabular}{ccccccc}
\hline \multirow{2}{*}{$\begin{array}{c}\text { Number of } \\
\text { Components }\end{array}$} & beta-s & beta-d & norm-s & norm-d & norm-cs & norm-cd \\
\hline 2 & 0.057 & 0.081 & 0.341 & 0.388 & 89.996 & 89.452 \\
3 & 0.033 & 0.040 & 0.129 & 0.161 & 70.309 & 69.975 \\
4 & 0.037 & 0.040 & 0.091 & 0.088 & 58.130 & 57.903 \\
5 & 0.028 & 0.034 & 0.044 & 0.047 & 35.771 & 35.542 \\
6 & 0.019 & 0.031 & 0.033 & 0.039 & 38.333 & 38.323 \\
\hline
\end{tabular}

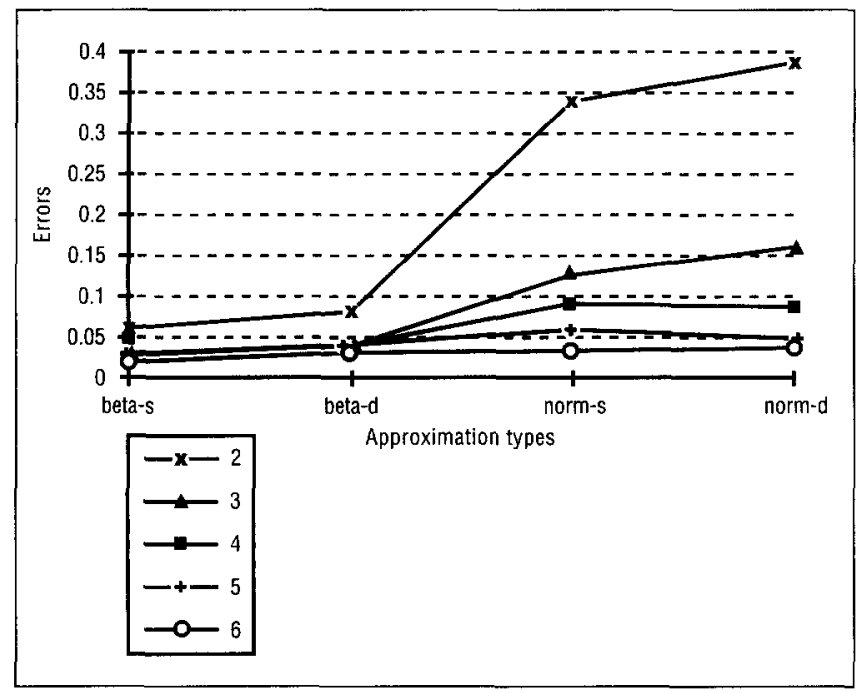

Figure 4

Plot of Approximation Errors Versus Approximation Types (Group III Distributions)

can handle the skewed distributions better than the normal distribution.

Other findings from the validation process of the BDAM are as follows:

- For all groups of distributions, approximation using a normal distribution with centered mean appeared inadequate.

- When the component number is larger than five, the approximation errors of beta and normal distributions (with sampled or derived parameters) are very close.

- Using the beta distribution with a sample mean and standard deviation appeared to be the best approximation method. However, it is less suitable in tolerance synthesis if combined with an optimization procedure.

- Comparing the least sum of squares errors, the BDAM using the beta distribution with derived parameters is a good choice. In most cases, errors of the BDAM are smaller than those of normal distributions.

\section{Application of the BDAM in Tolerance Analysis}

Tolerance analysis is a procedure that verifies the resultant tolerance by identifying the individual dimensions and stacking up those related tolerances. If design requirements are not met, tolerance values of individual components are adjusted and the resultant tolerance is recalculated. In Figure 5, two individual dimension distributions are shown on the lefthand side, while the resultant dimension distribution, which is the dimension of concern and is the summation of the two given individual dimensions, is illustrated on the right-hand side. The dashed line in Figure 5 indicates an unknown dimension distribution coming from a real manufacturing situation.

The distribution approximation method is applied to tolerance analysis. The component distributions are assumed to follow beta distributions and to be independent of each other. The calculations are conducted using the following procedures.

1. Identify $n$ related component dimensions, $X_{i} \pm \delta_{i}$ and $f_{i}, i=1,2, \ldots, n$, in the dimension chain. $f_{i}$ indicates the direction of $i$-th link in the dimension chain. Its value is either +1 or -1 , depending on the direction of the link.

2. Find the lower and upper specification limits, denoted $X_{i}{ }_{i}$ and $X^{u}{ }_{i}$, respectively, and their corresponding distribution parameters, $\alpha_{i}$ and $\beta_{i}$. $X_{i}^{i}$ and $X^{u}{ }_{i}$ can be obtained as follows:

$$
X_{i}{ }_{i}=X_{i}-\delta_{i} \quad X^{u_{i}}=X_{i}+\delta_{i}
$$

3. Apply the BDAM to find parameters of the resultant dimension distribution, $\alpha_{r}$ and $\beta_{r}$.

4. Find the lower and upper limits, $a$ and $b$ (see Figure 6), for the resultant dimension from the following equations:

$$
a=\sum_{i=1}^{n} f_{i} A_{i} \quad b=\sum_{i=1}^{n} f_{i} B_{i}
$$

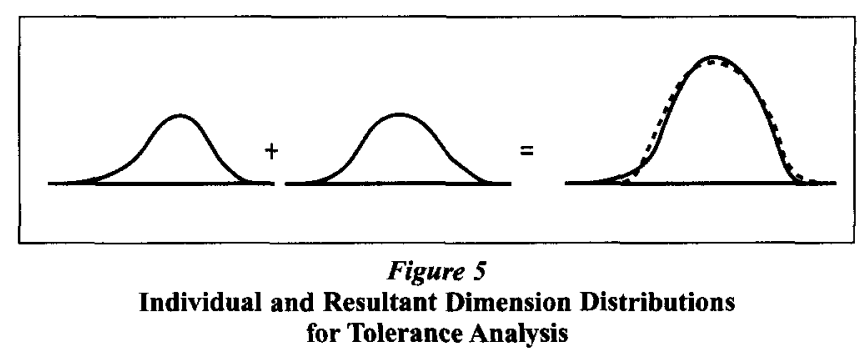




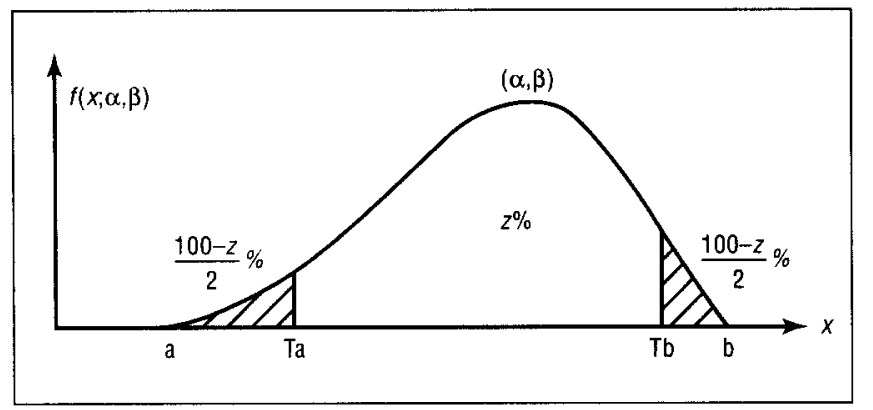

Figure 6

A Beta Distribution

where

$$
\begin{aligned}
& A_{i}=\left\{\begin{array}{l}
X_{i}^{l}, \text { if } f_{i}=+1 \\
X_{i}^{u}, \text { if } f_{i}=-1
\end{array}\right. \\
& B_{i}=\left\{\begin{array}{l}
X_{i}^{u}, \text { if } f_{i}=+1 \\
X_{i}^{l}, \text { if } f_{i}=-1
\end{array}\right.
\end{aligned}
$$

5. Calculate $T_{a}$ and $T_{b}$ using an inverse unit beta probability distribution function with $z \%$ confidence level and equal tail probability.

6. Obtain the resultant dimension and its tolerance: $X_{r} \pm \delta_{r}$

where $X_{r}=\frac{T_{a}+T_{b}}{2}$ and $\delta_{r}=\frac{T_{b}-T_{a}}{2}$.

\section{An Example}

A grinder head assembly (see Figure 7) is adopted from Lange ${ }^{14}$ as an example to illustrate the calculation of tolerance analysis. The assembly consists of 15 parts. For tolerance analysis, three major parts are considered: housing, cap, and shaft.

For a tolerance analysis problem, a dimension chain is formed and the related dimensions are identified. A dimension chain consists of two types of links: resultant link and component link. In the grinder head assembly example, the resultant link of the dimension chain is the dimension of protrusion on shaft shoulder $\left(X_{r} \pm \delta_{r}\right)$, and the component links are related dimensions from housing, cap, and shaft parts, denoted in Figure 6 as $X_{1} \pm \delta_{1}, X_{2} \pm \delta_{2}$, and $X_{3}$ $\pm \delta_{3}$, respectively. It is noted that, in this example, bilateral tolerance expression is utilized. In fact, the BDAM is able to handle cases of unilateral tolerance expression.

To analyze the dimension on the shaft shoulder, the dimension specification of related component links should be given. Table 4 shows the information

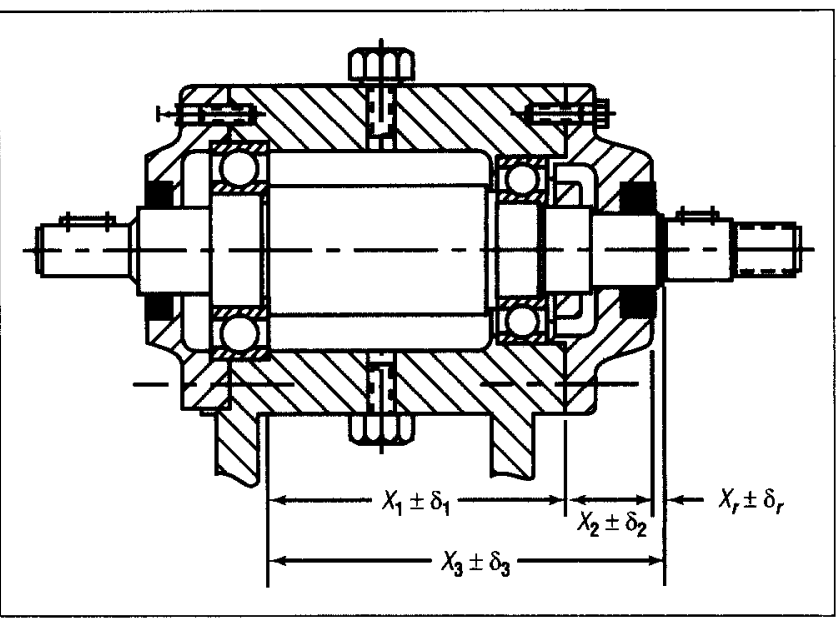

Figure 7

A Grinder Head Example

needed to carry out the tolerance analysis calculation. In Table 4, the nominal dimension, tolerance specification, and machining information for each part are given. For instance, the dimension on the housing of the grinder head is specified as $5.000 \pm$ 0.002 in. A process is selected to produce this dimension for the housing part, which determines the tolerancing specification and is modeled by a beta distribution with parameters $(\alpha, \beta)=(3.4,3.4)$. Parameters for each process can be obtained from machine accuracy and historical production data.

Applying the tolerance analysis procedures mentioned earlier, intermediate information can be obtained and is shown as follows: $a=0.088, b=$ $0.112, \alpha_{r}=11.721$, and $\beta_{r}=12.590$.

The inverse beta distribution function can be found in most commercial spreadsheet software program. The input to the inverse beta distribution function is a cumulative probability, shape parameters $(\alpha, \beta)$, and generalized distribution range $(a, b)$. The function returns the value of $x$ (see Figure 6 ), which is, in terms of tolerance analysis, the resultant dimension. If $99.73 \%$ assembly yield is assumed, then the result obtained from the BDAM is 0.09965

Table 4

Design and Manufacturing Information

\begin{tabular}{lcccc}
\hline $\begin{array}{c}\text { Assembly } \\
\text { Component } \\
(i)\end{array}$ & $\begin{array}{c}\text { Nominal } \\
\text { Dimension } \\
\left(X_{i}\right)\end{array}$ & $\begin{array}{c}\text { Unilateral } \\
\text { Tolerance } \\
\left(\delta_{i}\right)\end{array}$ & $\begin{array}{c}\text { Direction } \\
\text { Vector } \\
\left(f_{i}\right)\end{array}$ & $\begin{array}{c}\text { Beta } \\
\text { Parameters } \\
(\alpha, \beta)\end{array}$ \\
\hline (1) Housing & 5.000 & 0.002 & -1 & $(3.4,3.4)$ \\
(2) Cap & 1.500 & 0.005 & -1 & $(4.2,3.6)$ \\
(3) Shaft & 6.600 & 0.005 & +1 & $(3.1,4.5)$ \\
\hline
\end{tabular}


$\pm 0.00675 \mathrm{in}$. In other words, the dimension of protrusion on the shaft shoulder varies from 0.0926 in. to 0.1064 in.

\section{Conclusions}

A method called the beta distribution approximation method was developed. This method overcomes several difficulties of traditional normal distribution-based tolerance analysis approaches. It provides an avenue to apply tolerance analysis to real-world manufacturing problems.

Several conclusions came from this study:

- The beta distribution is appropriate to model manufacturing processes because of its distribution properties.

- The calculation of the BDAM is deterministic, which requires significantly less computation than that of a simulation method.

- The approximation errors from the BDAM are relatively small, and therefore, the BDAM provides a reliable method for approximating the unknown resultant distribution.

- The BDAM handles a small dimension chain well. When there is a small number of components in a dimension chain, the BDAM still provides a good analysis result.

- The BDAM can be applied to tolerance analysis because the resultant distribution can be found. This method can also be utilized to tolerance synthesis if combined with an optimization procedure.

\section{References}

1. O.R. Wade, Tolerance Control in Design and Manufacturing (New York: Industrial Press, 1967).

2. E.M. Mansoor, "The Application of Probability to Tolerances Used in Engineering Design," Proceedings of the Institute of Mechanical Engineers (v178, nl, 1963), pp29-51.

3. D.B. Parkinson, "Tolerancing of Component Dimensions in CAD," Computer-Aided Design (v16, n1, Jan. 1984), pp25-32.

4. W.H. Greenwood and K.W. Chase, "A New Tolerance Analysis Method for Designers and Manufacturers," Journal of Engineering for Industry (v109, May 1987), pp112-116.

5. M.F. Spotts, "Dimensioning Stacked Assemblies," Machine Design (v50, n9, 1978), pp60-63.
6. O. Bjorke, Computer Aided Tolerancing, 2nd ed. (New York: ASME Press, 1989).

7. Z. Dong and A. Soom, "Automatic Optimal Tolerance Design for Related Dimension Chains," Manufacturing Review (v3, n4, Dec. 1990), pp262-271.

8. Z. Dong, "Automatic Tolerance Analysis and Synthesis in CAD Environments," PhD dissertation (State University of New York at Buffalo, May 1989).

9. G.J. Hahn and S.S. Shapiro, Statistical Models in Engineering (New York: John Wiley \& Sons, 1967).

10. D.H. Evans, Probability and Its Applications for Engineers (New York: Marcel Dekker, 1992), p215.

11. C. Zhang, "Tolerance Analysis and Synthesis for Design and Manufacturing," PhD dissertation (Iowa City, IA: University of Iowa, Aug. 1993).

12. C. Ash, The Probability Tutoring Book (New York: IEEE, 1993), pp204-205.

13. Z. Wu, W.H. ElMaraghy, and H.A. ElMaraghy, "Evaluation of CostTolerance Algorithms for Design Tolerance Analysis and Synthesis," Manufacturing Review (v1, n3, Oct. 1988), pp 168-179.

14. C.J. Lange, Design Dimensioning with Computer Graphics Applications (New York: Marcel Dekker, 1984), p203.

\section{Authors' Biographies}

Dr. Shui-Shun Lin is an associate professor and chairman of the Department of Business Administration, National Chinyi Institute of Technology (Taiwan), where he founded an Integrated Commerce Automation Laboratory. His current research interests include tolerance analysis and synthesis, $\mathrm{CAD} / \mathrm{CAM}$, material handling systems, and logistics management.

Dr. Hsu-Pin (Ben) Wang is chairman of the Department of Industrial Engineering at FAMU-FSU College of Engineering and is the DOE Dr. Samuel P. Massie Chair of Excellence, funded by the US Department of Energy. His chief research interest is in applying analytical methods to the improvement of quality, productivity, and manufacturing competitiveness. Particular areas of interest are product/process design, predictive maintenance, and design of intelligent manufacturing systems. The author and coauthor of more than 70 refereed journal articles, Dr. Wang is an editorial board member of five technical journals in design and manufacturing. His research projects have been with a number of organizations, including the National Science Foundation, the National Center of Earthquake Engineering Research, ARPA, ONR, Army FCIM Office, General Motors, Caterpillar, Westinghouse, and British Petroleum. He has served on a number of NSF grants review panels and on a Natural Sciences and Engineering Research Council of Canada grants review committee.

Dr. Chun (Chuck) Zhang is an assistant professor of industrial engineering at FAMU-FSU College of Engineering, where he teaches courses in computer-integrated manufacturing and industrial robotics. His current research interests include geometric tolerancing and metrology, system integration with STEP, computer-aided process planning and composite materials processing. Dr. Zhang has published more than 20 refereed journal articles in the above areas. He has been a guest editor of a special issue on mechanical tolerancing and metrology for the International Journal of Engineering Design and Automation. 